\title{
An Unexpected Liver Nodule Following Gastric Endoscopic Submucosal Dissection
}

\author{
Tiago Leal $^{1}$, Joana Areal ${ }^{2}$, Aníbal Ferreira ${ }^{1}$
}

1) Gastroenterology Department, Hospital de Braga, Braga; 2) Pathology Department, Hospital de Braga, Braga, Portugal
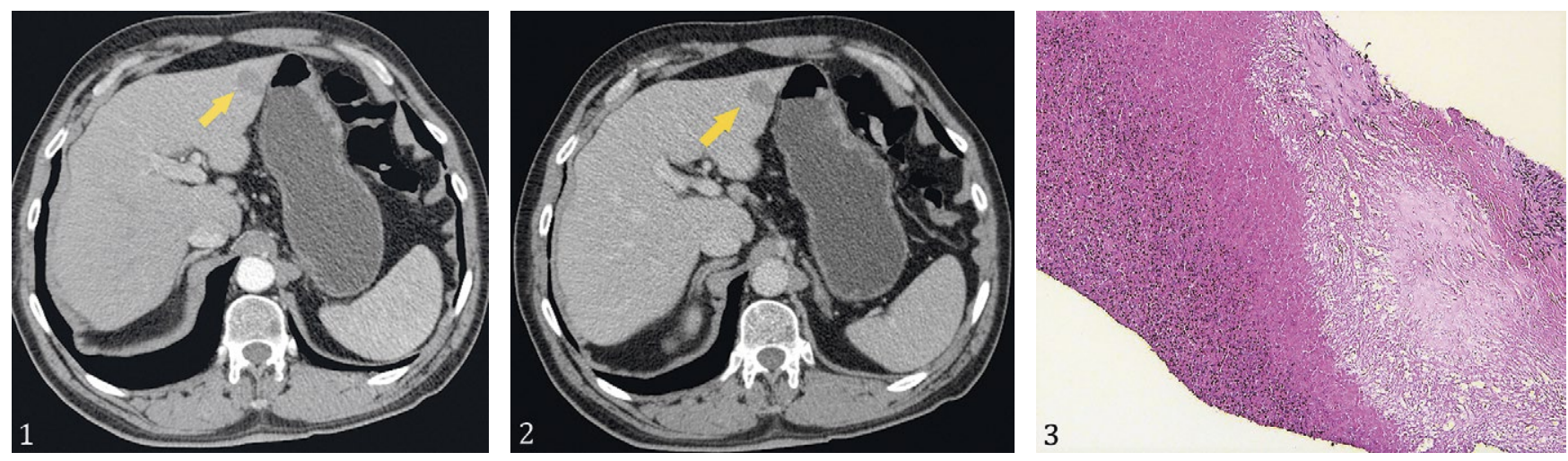

A 68-year-old patient underwent an endoscopic control following gastric endoscopic submucosal dissection (ESD), which showed a recurrent lesion at a previous ESD site. The $20 \mathrm{~mm}$ lesion, 0-IIb (Paris classification) in the lesser curvature of the gastric antrum was scheduled for removal by repeated ESD. However, the underlying fibrosis complicated the procedure to such an extent that piecemeal resection was necessary, being complemented with the application of argon-plasma coagulation. The histological examination reported intramucosal adenocarcinoma. A staging computed tomography (CT) was ordered, revealing two contiguous, hypoechoic and hypocaptant nodular lesions both in the arterial phase (Fig. 1) and the venous phase (Fig 2), in the liver (segment II) with 23 and $17 \mathrm{~mm}$, adjacent to the gastric wall. An ultrasound guided liver biopsy was performed, whose findings were compatible with a liver abscess (Fig. 3).

The CT findings were rather surprising. Albeit extremely unlikely, the possibility of liver metastasis in a patient with gastric (intramucosal) adenocarcinoma raised concern. There are few reports of liver abscesses after colorectal polypectomy $[1,2]$, but to the best of our knowledge this is the first case of a liver abscess following gastric ESD. The likely mechanism behind this finding could have been transmural injury during the ESD, similar to a post-polypectomy syndrome. In addition, the use of APC might have had a key role in this event.

Corresponding author: Tiago Leal, tiago.afleal@gmail.com

Conflicts of interest: None to declare.

\section{REFERENCES}

1. Farmer AD, Browett K, Rusius V, Bhalerao S, Anderson MR. Pyogenic liver abscess as a complication of sigmoid polypectomy. Endoscopy 2007;39(Suppl 1):E261-E261. doi:10.1055/s-2007-966552

2. Gross RG, Reiter B, Korsten MA. Pyogenic liver abscess complicating colonoscopic polypectomy. Gastrointest Endosc 2008;67:767768. doi:10.1016/j.gie.2007.08.028 